\title{
DNA hypomethylation of the Krüppel-like factor 11 (KLF11) gene promoter: a putative biomarker of depression comorbidity in panic disorder and of non-anxious depression?
}

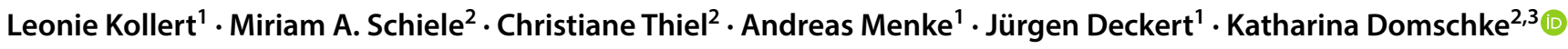

Received: 28 April 2020 / Accepted: 2 June 2020 / Published online: 10 June 2020

(c) The Author(s) 2020

\begin{abstract}
Panic disorder (PD) is one of the most common anxiety disorders and often occurs comorbidly with major depressive disorder (MDD). Altered methylation of the monoamine oxidase A (MAOA) gene has been implicated in the etiology of both PD and MDD. The Krüppel-like factor 11 (KLF11; alias TIEG2), an activating transcription factor of the MAOA gene, has been found to be increased in MDD, but has not yet been investigated in PD. In an effort to further delineate the effects of the KLF11-MAOA pathway in anxiety and affective disorders, KLF11 promoter methylation was analyzed via pyrosequencing of sodium bisulfite-treated DNA isolated from human peripheral blood in two independent samples of PD patients with or without comorbid MDD in a case-control design (sample 1: $N=120$ ) as well as MDD patients with and without anxious depression (sample 2: $N=170$ ). Additionally, in sample 1, KLF11 methylation was correlated with Beck Depression Inventory (BDI-II) scores. No overall association of KLF11 promoter methylation with PD was detected. However, PD patients with comorbid MDD showed significant hypomethylation relative to both healthy controls $(p=0.010)$ and PD patients without comorbid MDD $(p=0.008)$. Furthermore, KLF11 methylation was negatively correlated with BDI-II scores in PD patients $(p=0.013)$. MDD patients without anxious features showed nominally decreased KLF11 methylation in comparison to MDD patients with anxious depression $(p=0.052)$. The present results suggest $K L F 11$ promoter hypomethylation as a potential epigenetic marker of MDD comorbidity in PD or of non-anxious depression, respectively, possibly constituting a differential pathomechanism in anxiety and mood disorders.
\end{abstract}

Keywords KLF11 · TIEG2 - Epigenetics · Panic disorder · Major depressive disorder · TGFB-inducible early growth response protein 2

Leonie Kollert and Miriam A. Schiele have contributed equally to this work and should therefore both be considered first authors.

Katharina Domschke

katharina.domschke@uniklinik-freiburg.de

1 Department of Psychiatry, Psychosomatics and Psychotherapy, University of Würzburg, Würzburg, Germany

2 Department of Psychiatry and Psychotherapy, Medical Center-University of Freiburg, Faculty of Medicine, University of Freiburg, Freiburg, Germany

3 Center for Basics in Neuromodulation, Faculty of Medicine, University of Freiburg, Freiburg, Germany

\section{Introduction}

Panic disorder (PD) is one of the most common anxiety disorders, with lifetime prevalence rates of $2.1-4.7 \%$ (Baxter et al. 2014) and a 12-month prevalence of $1.8 \%$ (Goodwin et al. 2005). PD is characterized by sudden, unexpected and recurrent panic attacks, i.e., episodes of extreme fear (American Psychiatric Association 2013). PD is often comorbid with major depressive disorder (MDD; Gorman and Coplan 1996): Lifetime prevalence rates of comorbid MDD in PD are estimated at about 30-40\% (Fava et al. 2000; Lamers et al. 2011; Kessler et al. 2015), similar to the lifetime prevalence rates of comorbid MDD in the group of anxiety disorders as a whole (Kessler et al. 2006).

The monoamine oxidase A (MAOA) - metabolizing catecholamines such as norepinephrine and serotonin and being a target of potent antidepressants such as 
tranylcypromine-has been suggested as a key player in the pathogenesis of anxiety and mood disorders (for review see Ziegler and Domschke 2018). On an epigenetic level, MAOA hypomethylation has been reported in patients with PD (Domschke et al. 2012; Ziegler et al. 2016), acrophobia (Schiele et al. 2018), and MDD (Melas et al. 2013; Melas and Forsell 2015). Moreover, MAOA hypomethylation has been observed to be predictive of response to pharmacological treatment in MDD (Domschke et al. 2012) and to be modifiable by psychotherapeutic interventions in anxiety disorders (Ziegler et al. 2016; Schiele and Domschke 2018; Schiele et al. 2018; for review see Schiele et al. 2020).

Recent studies have highlighted the Krüppel-like factor 11 (KLF11; alias TGFB-Inducible Early Growth Response Protein 2 [TIEG2]) as a novel transcriptional activator of MAOA gene expression (for review see Duncan et al. 2012). KLF11 is a member of the Sp/KLF family of zinc finger transcription factors and regulates the transcription of neuronal genes by binding to distinct sequences within the target gene promoter region, triggering RNA polymerase II (Pol II)-mediated transcriptional initiation (Ou et al. 2004; Harris et al. 2015). The MAOA promoter contains four Sp1-binding sites which have been reported to be a target of KLF11. Accordingly, KLF11 transfection has been observed to result in a twofold increase in MAOA mRNA expression (Grunewald et al. 2012). Further support for a KLF11-MAOA pathway emerges from a study showing elevated KLF11 protein levels correlating with increased MAOA levels in postmortem brain samples of MDD patients (Harris et al. 2015).

Given these close links between KLF11 and MAOA function, the KLF11 (TIEG2) gene on chromosome 2p25.1 might constitute a prime, yet uninvestigated candidate gene in the panic disorder/MDD spectrum. Thus, in the present study, we for the first time examined KLF11 DNA methylation levels in (1) patients with panic disorder in a case-control design taking into account comorbidity with MDD, and (2) in an independent sample of patients with MDD by comparing symptom subtypes (i.e., anxious vs. non-anxious depression) in an effort to for the first time explore the role of KLF11 on an epigenetic level in anxiety and affective disorders.

\section{Materials and methods}

\section{Samples}

Sample 1 consisted of 60 Caucasian (for at least two preceding generations) patients with PD (47 females; age [mean $\pm \mathrm{SD}$ ]: $34.28 \pm 9.49$ years $)$ with $(N=28,46.6 \%)$ or without agoraphobia, and 60 healthy controls matched by age (mean \pm SD: $34.30 \pm 9.25$ years; $t=0.01, d f=118$, $p=0.992)$ and sex (47 females; $\left.\mathrm{X}^{2}=0.0, d f=1, p=1.0\right)$. PD diagnosis was confirmed by experienced clinical psychologists on the basis of a clinical interview according to DSMIV criteria (SCID-I; Wittchen et al. 1997). The presence of current comorbid axis I diagnoses other than bipolar disorder, psychotic disorders, current alcohol dependence, current abuse of or dependence on benzodiazepines and other psychoactive substances was tolerated if PD was the primary diagnosis (MDD: $N=28$; social anxiety disorder: $N=3$; specific phobias: $N=2$ ). Further exclusion criteria were current or past internal or neurological somatic illnesses, intake of any somatic medication, illegal drugs including cannabis (assessed by urine toxicology), pregnancy and excessive alcohol (> 15 glasses of alcohol per week) or nicotine ( $>20$ cigarettes per day) consumption. Thirty-four (56.7\%) patients were on stable psychiatric medication with selective serotonin reuptake inhibitors (SSRIs, $N=18$ ), serotoninnorepinephrine reuptake inhibitors (SNRIs, $N=5$ ), a noradrenergic and specific serotonergic antidepressant (NaSSA, $N=7$ ), tricyclic antidepressants (TCAs, $N=7$ ), pregabaline $(N=2)$, quetiapine $(N=2)$, or zopiclone $(N=1)$. No patient received monoamine oxidase inhibitors or valproate. Smoking status was assessed in the patient (smokers: $N=19$ ) and control groups $(N=18)$. In the control group, absence of current and/or lifetime DSM-IV mental axis I disorders was assessed using the Mini International Neuropsychiatric Interview (M.I.N.I.; Sheehan et al. 1998). The severity of depressive symptoms in patients was assessed using the 21-item Beck Depression Inventory (BDI-II; Hautziger et al. 2009). All participants were recruited at the Department of Psychiatry, University of Würzburg, Germany, within the Collaborative Research Centre SFB-TRR-58 'Fear, Anxiety, Anxiety Disorders', project C02.

Sample 2 comprised 170 Caucasian patients diagnosed with major depressive disorder (MDD; 98 females; age [mean $\pm \mathrm{SD}$ ]: $44.61 \pm 14.86$ years $)$ with $(\geq 7$ on the anxiety/somatization factor of the Hamilton Depression Rating Scale; cf. Fava et al. 2008; Domschke et al. 2010; $N=99$, $58.24 \%$ ) or without anxious depression. MDD diagnosis was determined by experienced clinical psychologists or psychiatrists based on medical records and a structured clinical interview according to DSM-IV criteria (SCID-I; Wittchen et al. 1997). Exclusion criteria were current obsessive-compulsive disorder, schizoaffective disorder, psychosis or dementia, and/or presence of substance abuse disorder or eating disorder currently or within the last 10 years. Smoking status was assessed in the overall sample (smokers: $N=52,30.59 \%) . N=135$ (79.4\%) patients received psychiatric medication with selective serotonin reuptake inhibitors (SSRIs, $N=48$ ), serotonin-norepinephrine reuptake inhibitors (SNRIs, $N=48$ ), a noradrenergic and specific serotonergic antidepressant (NaSSA, $N=36$ ), tricyclic antidepressants (TCAs, $N=26$ ), a noradrenaline and dopamine reuptake 
inhibitor (NDRI, $N=6)$, neuroleptics $(N=33)$, anticonvulsants $(N=12)$, benzodiazepines $(N=13)$, lithium $(N=7)$, or tianeptine $(N=1)$. No patient received monoamine oxidase inhibitors or valproate. All participants were recruited at the Department of Psychiatry, University of Würzburg, Germany.

This study was approved by the ethics committee of the University of Würzburg, Germany, and was conducted according to the ethical principles of the Helsinki Declaration. Written informed consent was obtained from all participants.

\section{KLF11 DNA methylation analysis}

Venous blood samples were collected from all participants using standardized EDTA tubes. DNA extractions from frozen whole blood were accomplished using either FlexiGene ${ }^{\circledR}$ DNA Kit (Qiagen, Hilden, Germany) or a standardized salting out procedure (Miller et al. 1988). After pre-analysis of a 313 bp-long KLF11 promoter region (chr2:10,182,891-10,183,204; GRCh37/hg19 Assembly, UCSC Genome Browser) by Sanger sequencing (LGC Genomics, Berlin, Germany) according to previous studies (e.g., Domschke et al. 2012; Ziegler et al. 2016; Schiele et al. 2018, 2019, 2020), a $72 \mathrm{bp}$ amplicon containing part of the KLF11 promoter (five CpGs) upstream of exon I (chr2:10,183,094-10,183,166; GRCh37/hg19 Assembly, UCSC Genome Browser) was chosen for further DNA methylation analyses, given that only these five $\mathrm{CpG}$ sites showed a considerable variance in their mean methylation level and thus seemed to be suitable for subsequent DNA methylation analyses by pyrosequencing.

Aliquots (500 ng) of isolated genomic DNA were treated with sodium bisulfite using the EpiTect ${ }^{\circledR} 96$ Bisulfite Kit (Qiagen, Hilden, Germany) according to the manufacturer's instructions and processed in randomized order to avoid possible batch effects. Non-methylated and fully methylated EpiTect ${ }^{\circledR}$ PCR Control DNA Sets (Qiagen, Hilden, Germany) were used as a control for successful bisulfite conversion.

The 72 bp amplicon $(10,183,094-10,183,166$; GRCh37/ hg19 Assembly, UCSC Genome Browser) was amplified via PCR according to a standard pyrosequencing PCR protocol (Qiagen, Hilden, Germany). The obtained DNA strand was sequenced and quantitatively analyzed by pyrosequencing using PyroMark ${ }^{\circledR}$ Q96 ID System (Qiagen, Hilden, Germany). All samples were tested in duplicate to check for run variability resulting in a mean methylation score for each $\mathrm{CpG}$ site as well as an individual standard deviation (SD) for each duplicate. For quality control, $\mathrm{SD}>0.1$ of each duplicate was used as exclusion criterion. Additionally, outliers ( $\geq 3^{*} \mathrm{SD}$ from mean methylation of respective $\mathrm{CpG}$ site) were defined as second exclusion criterion. In each sample,
$N=6$ participants had to be excluded from the reported analyses according to these criteria.

The obtained electropherograms were robustly readable for all five $\mathrm{CpG}$ sites, and $\mathrm{CpG}$ sites were numbered according to their GRCh37/hg19 Primary Assembly position: $\mathrm{CpG} 1=\operatorname{chr} 2: 10,183,094 ; \mathrm{CpG} 2=\operatorname{chr} 2: 10,183,106$; CpG3 $=\operatorname{chr} 2: 10,183,115 ; \quad$ ppG4 $=\operatorname{chr} 2: 10,183,125$; CpG5 = chr2:10,183,140 (all genomic locations according to GRCh37/hg19 Assembly, UCSC Genome Browser).

\section{Statistical analysis}

Differences in dimensional sample characteristics were tested by means of independent samples $t$ tests, and differences in categorical variables by means of Chi-square tests. KLF11 methylation (average methylation, single CpG sites) between PD patients and controls (sample 1) and between MDD patients with anxious and non-anxious depression (sample 2), respectively, was compared by means of non-parametric Kruskal-Wallis test. Correlations between KLF11 methylation and BDI-II scores in sample 1 were evaluated by means of Spearman correlations $(\rho)$. Since no associations between average KLF11 methylation and sex (sample 1: $t_{112}=1.056, p=0.293$; sample 2: $t_{162}=-0.137, p=0.891$ ), age (sample 1: $r=0.156 ; p=0.097$; sample 2: $r=0.054$; $p=0.495$ ), smoking status (sample $1: t_{112}=0.745, p=0.458$; sample 2: $t_{161}=1.213 ; p=0.227$ ), or medication intake (sample 1: $t_{57}=1.384, p=0.172$; sample 2: $t_{162}=-0.123$, $p=0.902$ ) were found in either sample, analyses were not controlled for these variables. The significance level was set at $p \leq 0.05$. Given the present proof-of-concept approach and high correlation between single $\mathrm{CpG}$ sites (data not shown), no correction for multiple testing was applied when analyzing single CpG sites and average KLF11 methylation (cf. Schiele et al. 2018).

\section{Results}

\section{Sample 1}

In the overall case-control comparison between PD patients and healthy controls, no significant differences in average methylation or in DNA methylation at single $\mathrm{CpG}$ sites was discerned (see Table 1). Secondary analyses comparing PD patients with and without comorbid MDD indicated significant group differences for average methylation ( $p=0.049)$ and methylation at CpG3 $(p=0.041)$. Follow-up tests revealed significantly decreased methylation in PD patients with comorbid MDD (PD + MDD; $N=27)$ regarding average $K L F 11$ methylation $(p=0.008)$ and $\mathrm{CpG}$ site 3 methylation $(p=0.006)$ compared to $\mathrm{PD}$ patients without comorbid MDD as well as compared to 
Table 1 KLF11 methylation levels in PD patients and matched healthy controls

\begin{tabular}{lrrl}
\hline CpG & \multicolumn{3}{l}{${\text { Sample } 1^{\mathrm{a}}}$} \\
\cline { 2 - 4 } & $\begin{array}{l}\text { PD patients } \\
(\text { mean } \pm \text { SE) }\end{array}$ & \multicolumn{1}{l}{ Controls (mean \pm SE) } & Statistics $^{\mathrm{b}}$ \\
& $N=57$ & & \\
\hline Average & $11.29 \pm 0.45$ & $11.88 \pm 0.64$ & $z=-0.25 ; p=0.803$ \\
1 & $2.55 \pm 0.14$ & $2.83 \pm 0.21$ & $z=-0.60 ; p=0.552$ \\
2 & $11.67 \pm 0.55$ & $11.93 \pm 0.65$ & $z=-0.10 ; p=0.919$ \\
3 & $24.01 \pm 0.91$ & $25.38 \pm 1.30$ & $z=-0.33 ; p=0.740$ \\
4 & $8.90 \pm 0.38$ & $9.04 \pm 0.46$ & $z=-0.00 ; p=0.998$ \\
5 & $9.29 \pm 0.35$ & $9.46 \pm 0.39$ & $z=-0.06 ; p=0.956$ \\
\hline
\end{tabular}

${ }^{\text {a }} P D$ panic disorder

${ }^{\mathrm{b}} p$ values from non-parametric Mann-Whitney $U$ test are reported with average DNA methylation or methylation at the respective single $\mathrm{CpG}$ sites as dependent variable and group (PD patients vs. healthy controls). Mean methylation given in \%; SE standard error of the mean

healthy controls, again regarding average methylation $(p=0.010)$ and methylation at $\mathrm{CpG}$ site $3(p=0.006)$ (see Table 2, Fig. 1). Conversely, PD patients without comorbid MDD diagnosis (PDonly; $N=30$ ) did not differ from healthy controls with regard to KLF11 average methylation and methylation at single $\mathrm{CpG}$ sites (all $p>0.05$; see Table 2).

On a dimensional level, a negative correlation of $K L F 11$ average methylation with BDI-II scores was observed in the patient group $(\rho=-0.336 ; p=0.013$; see Fig. 2).

\section{Sample 2}

Significant differences in KLF11 DNA methylation were observed between MDD patients with and without anxious depression at $\mathrm{CpG}$ site $1(p=0.041)$ and, on a nominally significant level, with regard to average methylation $(p=0.052)$ and methylation at $\mathrm{CpG}$ sites 2-4 ( $p=0.053-0.081$; see Table 3; Fig. 3), with patients with non-anxious depression showing decreased KLF11 methylation.

\section{Discussion}

In the present study, patients with PD and comorbid MDD displayed hypomethylation of the KLF11 (TIEG2) promoter region in comparison to a matched healthy control group as well as to PD patients without comorbid MDD. Correspondingly, a negative correlation of KLF11 DNA methylation with dimensional symptoms of depression (as evaluated via the BDI-II) was observed in the PD patient group. Furthermore, in an independent sample of MDD patients, MDD patients without anxious features showed nominally significantly decreased KLF11 methylation levels as compared to MDD patients with anxious depression.

The present results for the first time demonstrating $K L F 11$ promoter hypomethylation-presumably leading to increased KLF11 gene expression (cf. Suzuki and Bird 2008) and, consequently, an overexpression of the MAOA gene (Grunewald et al. 2012)—in depression-related phenotypes are in line with a previous finding of increased KLF11 and MAOA levels in MDD patients compared to healthy controls (Harris et al. 2015) and with studies reporting MAOA hypomethylation in female patients with MDD (Melas et al.

Table 2 KLF11 methylation levels in PD patients with and without comorbid MDD and matched healthy controls

\begin{tabular}{|c|c|c|c|c|c|c|c|}
\hline \multirow[t]{2}{*}{$\mathrm{CpG}$} & \multicolumn{7}{|l|}{ Sample $1^{\mathrm{a}}$} \\
\hline & $\begin{array}{l}\text { Pdonly } \\
(\text { mean } \pm \mathrm{SE}) \\
N=30\end{array}$ & $\begin{array}{l}\mathrm{PD}+\mathrm{MDD} \\
(\text { mean } \pm \mathrm{SE}) \\
N=27\end{array}$ & $\begin{array}{l}\text { Controls } \\
(\text { mean } \pm \mathrm{SE}) \\
N=57\end{array}$ & Statistics $^{b}$ & $\begin{array}{l}\text { PDonly vs. } \\
\text { controls }^{\mathrm{c}}\end{array}$ & $\begin{array}{l}\text { PD+MDD vs. } \\
\text { controls }\end{array}$ & $\begin{array}{l}\text { PDonly vs. } \\
\text { PD + MDD }\end{array}$ \\
\hline Average & $12.22 \pm 0.61$ & $10.25 \pm 0.62$ & $11.88 \pm 0.63$ & $\mathrm{H}=6.04 ; p=\mathbf{0 . 0 4 9} *$ & $z=-1.94$ & $\begin{aligned} 3 z & =-2.73 \\
p & =\mathbf{0 . 0 1 0} * *\end{aligned}$ & $\begin{array}{l}z=-2.65 \\
p=\mathbf{0 . 0 0 8} * *\end{array}$ \\
\hline 1 & $2.79 \pm 0.20$ & $2.29 \pm 0.18$ & $2.84 \pm 0.21$ & $\mathrm{H}=4.35 ; p=0.113$ & - & - & - \\
\hline 2 & $12.72 \pm 0.76$ & $10.51 \pm 0.73$ & $11.93 \pm 0.65$ & $\mathrm{H}=5.06 ; p=0.080$ & - & - & - \\
\hline 3 & $25.99 \pm 1.22$ & $21.81 \pm 1.25$ & $25.38 \pm 1.30$ & $\mathrm{H}=6.39 ; p=\mathbf{0 . 0 4 1} *$ & $z=-1.93$ & $\begin{aligned} 4 z & =-2.56 \\
p & =\mathbf{0 . 0 0 6} * *\end{aligned}$ & $\begin{array}{l}z=-2.73 \\
p=0.006 * *\end{array}$ \\
\hline 4 & $9.63 \pm 0.51$ & $8.09 \pm 0.54$ & $9.04 \pm 0.46$ & $\mathrm{H}=5.26 ; p=0.072$ & - & - & - \\
\hline 5 & $9.95 \pm 0.47$ & $8.56 \pm 0.49$ & $9.46 \pm 0.39$ & $\mathrm{H}=5.59 ; p=0.061$ & - & - & - \\
\hline
\end{tabular}

${ }^{\text {a }} P D$ panic disorder, $M D D$ major depressive disorder

${ }^{\mathrm{b}} p$ values from non-parametric Kruskal-Wallis test are reported with average DNA methylation or methylation at the respective single CpG sites as dependent variable and group (healthy controls vs. PDonly patients vs. PD + MDD patients)

${ }^{\mathrm{c}} z$ and $p$ values from post hoc tests (Mann-Whitney $U$ test) are reported. Bold: significant results: *significant at $p \leq 0.05$; **significant at $p \leq 0.01$; mean methylation given in $\%$; SE standard error of the mean 


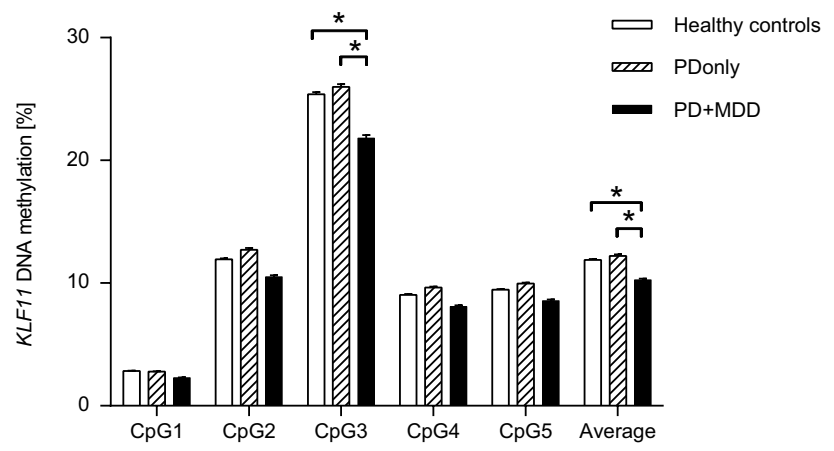

Fig. 1 KLF11 DNA methylation in panic disorder patients with or without comorbid major depressive disorder and matched healthy controls. $P D$ panic disorder, $M D D$ major depressive disorder, Controls healthy controls without any anxiety disorder, PDonly PD patients without comorbid MDD, $P D+M D D$ PD patients with comorbid MDD; * $p \leq 0.05$

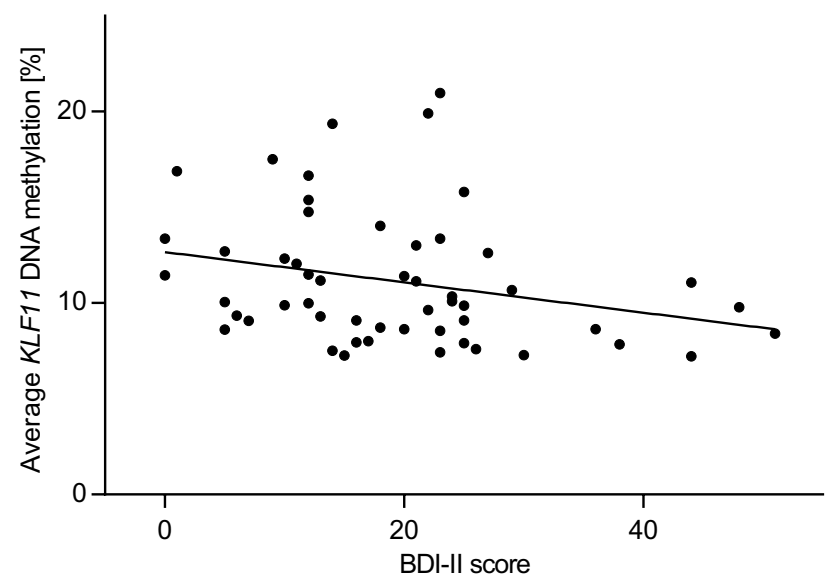

Fig. 2 KLF11 DNA methylation and dimensional depression in panic disorder. Negative correlation between average KLF11 DNA methylation and Beck Depression Inventory (BDI-II) scores in 54 patients with panic disorder $(\rho=-0.336, p=0.013)$

2013; Melas and Forsell 2015). The present findings, however, do not support a role of KLF11 methylation in PD. Thus, against the theoretical background of a common biological trunk shared by anxiety and mood disorders, KLF11 methylation could be instrumental in guiding the differentiation of the clinical phenotype toward depression- rather than panic-related symptoms.

Interestingly, the results of this study mirror a recently published finding of serotonin transporter gene (SLC6A4) promoter hypermethylation in PD patients with comorbid MDD relative to healthy controls, but not in PD per se (Schiele et al. 2019). Consequently, future studies might want to explore the biochemical relationship between KLF11 and SLC6A4 function in the context of MDD, especially given that the $S L C 6 A 4$ promotor region has
Table 3 KLF11 DNA methylation in MDD patients with or without anxious depression

\begin{tabular}{|c|c|c|c|}
\hline \multirow[t]{2}{*}{$\mathrm{CpG}$} & \multicolumn{3}{|l|}{ Sample $2^{\mathrm{a}}$} \\
\hline & $\begin{array}{l}\text { Anxious } \\
\text { depression } \\
\text { (mean } \pm \mathrm{SE}) \\
N=98\end{array}$ & $\begin{array}{l}\begin{array}{l}\text { Non-anxious } \\
\text { depression } \\
\text { (mean } \pm \mathrm{SE} \text { ) }\end{array} \\
N=66\end{array}$ & Statistics $^{\mathrm{b}}$ \\
\hline Average & $12.28 \pm 0.37$ & $11.23 \pm 0.41$ & $z=-1.95 ; p=0.052$ \\
\hline 1 & $3.07 \pm 0.12$ & $2.70 \pm 0.13$ & $\begin{array}{l}z=-2.04 \\
p=\mathbf{0 . 0 4 1 *}\end{array}$ \\
\hline 2 & $12.23 \pm 0.41$ & $11.20 \pm 0.46$ & $z=-1.75 ; p=0.081$ \\
\hline 3 & $25.46 \pm 0.70$ & $23.41 \pm 0.80$ & $z=-1.75 ; p=0.079$ \\
\hline 4 & $10.13 \pm 0.35$ & $9.18 \pm 0.38$ & $z=-1.77 ; p=0.077$ \\
\hline 5 & $10.51 \pm 0.31$ & $9.64 \pm 0.33$ & $z=-1.94 ; p=0.053$ \\
\hline
\end{tabular}

${ }^{a} M D D$ major depressive disorder, anxious depression 98 patients with anxious depression (anxiety/somatization factor of the Hamilton Depression Rating Scale $\geq 7$ ), non-anxious depression 66 patients without anxious depression (anxiety/somatization factor of the Hamilton Depression Rating Scale $<7$ ).

${ }^{\mathrm{b}} p$ values from non-parametric Mann-Whitney $U$ test are reported with average DNA methylation or methylation at the respective single $\mathrm{CpG}$ sites as dependent variable and group (anxious vs. non-anxious MDD patients)

Bold: significant results: *significant at $p \leq 0.05$; mean methylation given in \%; $S E$ standard error of the mean

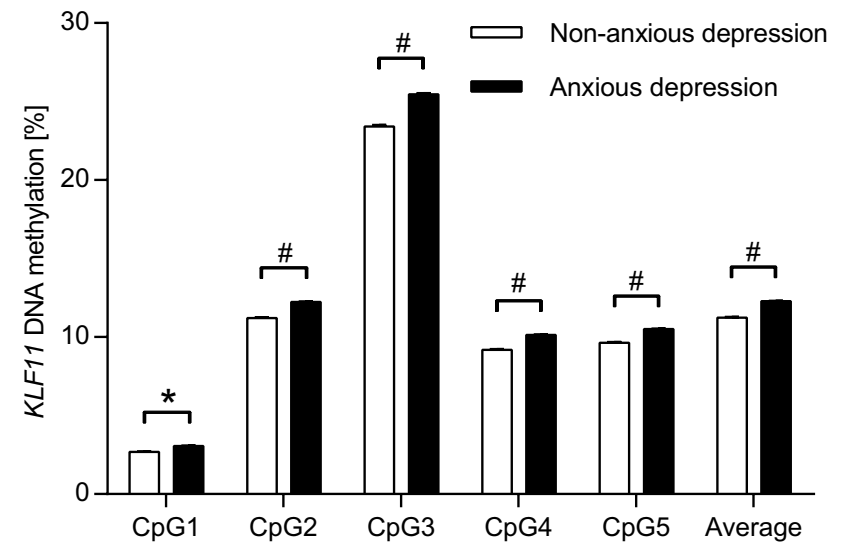

Fig. 3 KLF11 DNA methylation in MDD patients with or without anxious depression. MDD: major depressive disorder; anxious depression: 98 patients with anxious depression (anxiety/somatization factor of the Hamilton Depression Rating Scale $\geq 7$ ); non-anxious depression: 66 patients without anxious depression (anxiety/somatization factor of the Hamilton Depression Rating Scale $<7)$; ${ }^{\#} p \leq 0.1$; $* p \leq 0.05$

been shown to contain Sp1 binding sites (Bengel et al. 1997; Heils et al. 1998), which might be targeted by KLF11 (cf. Grunewald et al. 2012).

The present findings should be interpreted in light of some limitations and might inspire future research efforts. 
For example, replication in independent, larger samples is warranted due to the relatively small group sizes of PD patients with and without comorbid depression, or MDD patients with and without anxious features, respectively, to confirm KLF11 hypomethylation as a potential selective differential diagnostic marker of MDD comorbidity in PD or non-anxious MDD. Given that MDD is highly comorbid not only with PD but also with other anxiety and mental disorders, the potential of KLF11 methylation status as a diagnostic marker to separate MDD diagnosis from other diagnoses should be explored in future studies. This would require a direct comparison with other comorbidity profiles, as well as comorbidity of MDD within other anxiety or mental disorders and a direct comparison of a PD sample with an MDD sample (i.e., PD with MDD vs. MDD only, PD without MDD vs. MDD only). Additionally, since no control group was available for the MDD sample (sample 2), the observed differences of KLF11 methylation in MDD patients with or without anxious depression cannot be clearly ascribed to the effects of anxious symptoms. Moreover, longitudinal studies would help to elucidate whether the observed KLF11 hypomethylation constitutes a trait or state marker of MDD. Despite the fact that in the present study PD patients and controls were matched for sex, age and smoking status, additional potentially confounding factors such as environmental exposure (cf. Domschke et al. 2012) cannot be excluded. Therefore, investigation of the impact of life events on KLF11 methylation levels applying an (epi)gene-environment approach would pose a promising future research direction, particularly given previous evidence for a strong influence of stress on KLF11 expression (Grunewald et al. 2012; Harris et al. 2015). In addition, although no statistically significant influence of medication status on KLF11 methylation was observed in either sample, potentially confounding effects of psychiatric medication on KLF11 methylation cannot be fully excluded given that 57\% (sample 1) or $79 \%$ (sample 2), respectively, of patients were on stable medication at the time of testing. Also, next to the differential effects of KLF11 methylation on categorical disorder phenotypes, future studies may want to probe the association of KLF11 methylation with symptom subtypes/ subgroups as well as with disorder-specific intermediate phenotypes, and differential DNA methylation markers of panic disorder and MDD should be explored on an epigenomewide level by means of epigenome-wide association studies (EWAS) (cf. Shimada-Sugimoto et al. 2017; Iurato et al. 2017; Shimada et al. 2018; Ziegler et al. 2019). Although pyrosequencing is currently considered the gold standard of DNA methylation analysis with a sensitivity to reliably detect differences as small as 5\% (e.g., Dejeux et al. 2009; Migheli et al. 2013; Poulin et al. 2018), low methylation percentages $(<10 \%)$ may be confounded by unsystematic noise. In the present study, CpGs 2 and 3 achieved average methylation levels $>10 \%$ or $>20 \%$, respectively, and might therefore constitute regions of particular interest for further analyses. For instance, analysis of a restricted target region of CpGs 2 and 3 utilizing the transcription factor-binding site prediction tool PROMO (Messeguer et al. 2002) identified the binding site of transcription factor TFI-II, a ubiquitously expressed protein, involved in growth factor signaling (Roy et al. 2007), to be located at CpG3, thus constituting a putative transcription factor whose binding affinity at this region could be affected by DNA methylation. Thus, future functional approaches may want to experimentally confirm a differential influence of KLF11 methylation on TFI-II binding. Finally, DNA methylation was measured in peripheral blood samples. Given that epigenetic processes can act in a cell type-specific way, a cofounding effect due to potential variation in white blood cell composition between patients and controls cannot be excluded. Furthermore, epigenetic analyses in peripheral biomaterial such as blood does not allow for direct conclusions regarding methylation levels in brain tissue. Nevertheless, animal studies intraindividually comparing peripheral and central methylation or studies comparing peripheral methylation with central activity of the respective metabolite in humans using positron emission tomography (PET) have provided some evidence for DNA methylation levels measured in blood as a proxy for central processes (e.g., Murphy et al. 2005; Nohesara et al. 2011; Shumay et al. 2012). Also, when performing an in silico analysis of the correlation between blood and brain DNA methylation levels at the investigated KLF11 region using the online available "Blood Brain DNA Methylation Comparison Tool" (Hannon et al. 2015), peripheral DNA methylation of both CpG1 (cg22541755) and CpG3 (cg20702913) was positively correlated with prefrontal cortex methylation $(r \geq 0.332, p \leq 0.004)$. Analysis of further KLF11 CpGs was precluded by the fact that only CpGs 1 and 3 are available in this search tool based on Illumina $450 \mathrm{~K}$ array data.

In conclusion, the present pilot data suggest KLF11 promoter hypomethylation as a potential epigenetic marker of MDD comorbidity in PD or non-anxious depression, respectively, possibly contributing to a differential pathomechanism of anxiety and mood disorders. In synopsis with previous findings implicating KLF11 in depressionrelated phenotypes (Harris et al. 2015), this finding is of mechanistic relevance and might foster research efforts into exploring innovative therapeutic approaches targeting this pathway in the treatment of major depression.

Acknowledgements Open Access funding provided by Projekt DEAL. We gratefully acknowledge the support in patient recruitment and characterization by Dr. S. Stonawski, Dr. A. Gajewska, C. Wurst and M. Mahr as well as the skillful technical assistance by C. Gagel.

Author contributions KD and JD designed the study. KD, JD and AM supervised sample recruitment. LK and CT designed the methylation 
experiments that were performed by LK under the supervision of CT and KD. LK managed the literature searches and undertook the statistical analyses under the supervision of MAS. LK and MAS wrote the first draft of the manuscript. All authors contributed to and have approved the final manuscript.

Funding Funding for this study was provided by the German Research Foundation (DFG)-project no. 44541416-TRR 58, subprojects C02 and Z02 (to KD and JD) and a Grant by the Dr. Robert Pfleger Stiftung (to $\mathrm{KD}$ ).

\section{Compliance with ethical standards}

Conflicts of interest KD is a member of the Janssen Pharmaceuticals, Inc. Steering Committee Neurosciences. AM has received speaker honoraria from Vivantes AVK, MEDICE, Recordati and Servier. JD reports funding by the EU to P1vital and the Bavarian State Government to BioVariance. All other authors report no potential conflicts of interest.

Ethics approval This study was approved by the ethics committee of the University of Würzburg, Germany, and was conducted according to the ethical principles of the Helsinki Declaration.

Consent to participate Written informed consent was obtained from all participants.

Open Access This article is licensed under a Creative Commons Attribution 4.0 International License, which permits use, sharing, adaptation, distribution and reproduction in any medium or format, as long as you give appropriate credit to the original author(s) and the source, provide a link to the Creative Commons licence, and indicate if changes were made. The images or other third party material in this article are included in the article's Creative Commons licence, unless indicated otherwise in a credit line to the material. If material is not included in the article's Creative Commons licence and your intended use is not permitted by statutory regulation or exceeds the permitted use, you will need to obtain permission directly from the copyright holder. To view a copy of this licence, visit http://creativecommons.org/licenses/by/4.0/.

\section{References}

American Psychiatric Association (2013) Diagnostic and statistical manual of mental disorders. American Psychiatric Association Publishing, Washington

Baxter AJ, Vos T, Scott KM et al (2014) The global burden of anxiety disorders in 2010. Psychol Med 44:2363-2374. https://doi. org/10.1017/S0033291713003243

Bengel D, Heils A, Petri S et al (1997) Gene structure and 5'-flanking regulatory region of the murine serotonin transporter. Mol Brain Res 44:286-292. https://doi.org/10.1016/s0169-328x(96)00234-3

Dejeux E, El Abdalaoui H, Gut IG, Tost J (2009) Identification and quantification of differentially methylated loci by the pyrosequencing technology. Methods Mol Biol 507:189-205. https:// doi.org/10.1007/978-1-59745-522-0_15

Domschke K, Deckert J, Arolt V, Baune BT (2010) Anxious versus non-anxious depression: difference in treatment outcome. J Psychopharmacol 24:621-622. https://doi.org/10.1177/0269881108 097723

Domschke K, Tidow N, Kuithan H et al (2012) Monoamine oxidase A gene DNA hypomethylation-a risk factor for panic disorder? Int
J Neuropsychopharmacol 15:1217-1228. https://doi.org/10.1017/ S146114571200020X

Duncan J, Johnson S, Ou X-M (2012) Monoamine oxidases in major depressive disorder and alcoholism. Drug Discov Ther 6:112-122. https://doi.org/10.5582/ddt.2012.v6.3.112

Fava M, Rankin MA, Wright EC et al (2000) Anxiety disorders in major depression. Compr Psychiatry 41:97-102. https://doi. org/10.1016/S0010-440X(00)90140-8

Fava M, Rush AJ, Alpert JE et al (2008) Difference in treatment outcome in outpatients with anxious versus nonanxious depression: a STAR*D report. Am J Psychiatry 165:342-351. https://doi. org/10.1176/appi.ajp.2007.06111868

Goodwin RD, Faravelli C, Rosi S et al (2005) The epidemiology of panic disorder and agoraphobia in Europe. Eur Neuropsychopharmacol 15:435-443. https://doi.org/10.1016/j.euron euro.2005.04.006

Gorman JM, Coplan JD (1996) Comorbidity of depression and panic disorder. J Clin Psychiatry 57(Suppl 10):34-43

Grunewald M, Johnson S, Lu D et al (2012) Mechanistic role for a novel glucocorticoid-KLF11 (TIEG2) protein pathway in stress-induced monoamine oxidase A expression. J Biol Chem 287:24195-24206. https://doi.org/10.1074/jbc.M112.373936

Hannon E, Lunnon K, Schalkwyk L, Mill J (2015) Interindividual methylomic variation across blood, cortex, and cerebellum: implications for epigenetic studies of neurological and neuropsychiatric phenotypes. Epigenetics 10:1024-1032. https:// doi.org/10.1080/15592294.2015.1100786

Harris S, Johnson S, Duncan JW et al (2015) Evidence revealing deregulation of the KLF11-MAO A pathway in association with chronic stress and depressive disorders. Neuropsychopharmacology 40:1373-1382. https://doi.org/10.1038/npp.2014.321

Hautziger M, Keller F, Kühner F (2009) BDI-II-Beck-depressions-inventar. Hogrefe, Göttingen

Heils A, Wichems C, Mössner R et al (1998) Functional characterization of the murine serotonin transporter gene promoter in serotonergic raphe neurons. J Neurochem 70:932-939. https:// doi.org/10.1046/j.1471-4159.1998.70030932.x

Iurato S, Carrillo-Roa T, Arloth J et al (2017) DNA methylation signatures in panic disorder. Transl Psychiatry 7:1-10. https:// doi.org/10.1038/s41398-017-0026-1

Kessler RC, Chiu WT, Jin R et al (2006) The epidemiology of panic attacks, panic disorder, and agoraphobia in the National Comorbidity Survey Replication. Arch Gen Psychiatry 63:415-424. https://doi.org/10.1001/archpsyc.63.4.415

Kessler RC, Sampson NA, Berglund P et al (2015) Anxious and nonanxious major depressive disorder in the World Health Organization World Mental Health Surveys. Epidemiol Psychiatr Sci 24:210-226. https://doi.org/10.1017/S2045796015000189

Lamers F, van Oppen P, Comijs HC et al (2011) Comorbidity patterns of anxiety and depressive disorders in a large cohort study: The Netherlands Study of Depression and Anxiety (NESDA). J Clin Psychiatry 72:341-348. https://doi.org/10.4088/JCP.10m06 176blu

Melas PA, Forsell Y (2015) Hypomethylation of MAOA's first exon region in depression: a replication study. Psychiatry Res 226:389391. https://doi.org/10.1016/J.PSYCHRES.2015.01.003

Melas PA, Wei Y, Wong CCY et al (2013) Genetic and epigenetic associations of MAOA and NR3C1 with depression and childhood adversities. Int J Neuropsychopharmacol 16:1513-1528. https:// doi.org/10.1017/S1461145713000102

Messeguer X, Escudero R, Farré D et al (2002) PROMO: detection of known transcription regulatory elements using species-tailored searches. Bioinformatics 18:333-334. https://doi.org/10.1093/ bioinformatics/18.2.333

Migheli F, Stoccoro A, Coppedè F et al (2013) Comparison study of MS-HRM and pyrosequencing techniques for quantification of 
APC and CDKN2A gene methylation. PLoS ONE 8:e52501. https ://doi.org/10.1371/journal.pone.0052501

Miller SA, Dykes DD, Polesky HF (1988) A simple salting out procedure for extracting DNA from human nucleated cells. Nucleic Acids Res 16:1215. https://doi.org/10.1093/nar/16.3.1215

Murphy BC, O'Reilly RL, Singh SM (2005) Site-specific cytosine methylation in S-COMT promoter in 31 brain regions with implications for studies involving schizophrenia. Am J Med Genet Part B Neuropsychiatr Genet 133B:37-42. https://doi.org/10.1002/ ajmg.b.30134

Nohesara S, Ghadirivasfi M, Mostafavi S et al (2011) DNA hypomethylation of MB-COMT promoter in the DNA derived from saliva in schizophrenia and bipolar disorder. J Psychiatr Res 45:1432-1438. https://doi.org/10.1016/j.jpsychires.2011.06.013

Ou X-M, Chen K, Shih JC (2004) Dual functions of transcription factors, transforming growth factor-beta-inducible early gene (TIEG) 2 and Sp3, are mediated by CACCC element and Sp1 sites of human monoamine oxidase (MAO) B gene. J Biol Chem 279:21021-21028. https://doi.org/10.1074/jbc.M312638200

Poulin M, Zhou JY, Yan L, Shioda T (2018) Pyrosequencing methylation analysis. Methods Mol Biol 1856:283-296. https://doi. org/10.1007/978-1-4939-8751-1_17

Roy AL (2007) Signal-induced functions of the transcription factor TFII-I. Biochim Biophys Acta Gene Struct Express 1769:613621. https://doi.org/10.1016/j.bbaexp.2007.10.002

Schiele MA, Domschke K (2018) Epigenetics at the crossroads between genes, environment and resilience in anxiety disorders. Genes Brain Behav 17:e12423. https://doi.org/10.1111/gbb.12423

Schiele MA, Gottschalk MG, Domschke K (2020) The applied implications of epigenetics in anxiety, affective and stress-related disorders-a review and synthesis on psychosocial stress, psychotherapy and prevention. Clin Psychol Rev 77:101830. https:// doi.org/10.1016/j.cpr.2020.101830

Schiele MA, Kollert L, Lesch KP et al (2019) Hypermethylation of the serotonin transporter gene promoter in panic disorder-epigenetic imprint of comorbid depression? Eur Neuropsychopharmacol 29:1161-1167. https://doi.org/10.1016/j.euroneuro.2019.07.131

Schiele MA, Ziegler C, Kollert L et al (2018) Plasticity of functional MAOA gene methylation in Acrophobia. Int J Neuropsychopharmacol 21:822-827. https://doi.org/10.1093/ijnp/pyy050

Sheehan DV, Lecrubier Y, Sheehan KH et al (1998) The Mini-International Neuropsychiatric Interview (MINI): the development and validation of a structured diagnostic psychiatric interview for DSM-IV and ICD-10. J Clin Psychiatry 59(Suppl 20):22-33

Shimada-Sugimoto M, Otowa T, Miyagawa T et al (2017) Epigenomewide association study of DNA methylation in panic disorder. Clin Epigenetics 9:6. https://doi.org/10.1186/s13148-016-0307-1

Shimada M, Otowa T, Miyagawa T et al (2018) An epigenome-wide methylation study of healthy individuals with or without depressive symptoms. J Hum Genet 63:319-326. https://doi.org/10.1038/ s10038-017-0382-y

Shumay E, Logan J, Volkow ND, Fowler JS (2012) Evidence that the methylation state of the monoamine oxidase A (MAOA) gene predicts brain activity of MAO A enzyme in healthy men. Epigenetics 7:1151-1160. https://doi.org/10.4161/epi.21976

Suzuki MM, Bird A (2008) DNA methylation landscapes: provocative insights from epigenomics. Nat Rev Genet 9:465-476. https://doi. org/10.1038/nrg2341

Wittchen H-U, Wunderlich U, Gruschwitz S, Zaudig M (1997) SKID I. Strukturiertes Klinisches Interview für DSM-IV. Achse I: Psychische Störungen. Interviewheft und Beurteilungsheft. Eine deutschsprachige, erweiterte Bearb. d. amerikanischen Originalversion des SKID I. Hogrefe, Göttingen

Ziegler C, Domschke K (2018) Epigenetic signature of MAOA and MAOB genes in mental disorders. J Neural Transm 125:15811588. https://doi.org/10.1007/s00702-018-1929-6

Ziegler C, Grundner-Culemann F, Schiele MA et al (2019) The DNA methylome in panic disorder: a case-control and longitudinal psychotherapy-epigenetic study. Transl Psychiatry 9:1-11. https ://doi.org/10.1038/s41398-019-0648-6

Ziegler C, Richter J, Mahr M et al (2016) MAOA gene hypomethylation in panic disorder-reversibility of an epigenetic risk pattern by psychotherapy. Transl Psychiatry 6:e773. https://doi. org/10.1038/tp.2016.41

Publisher's Note Springer Nature remains neutral with regard to jurisdictional claims in published maps and institutional affiliations. 